\title{
The VIMOS-VLT Deep Survey
}

\section{The evolution of galaxy clustering per spectral type to $z \simeq 1.5^{\star}$}

\author{
B. Meneux ${ }^{1}$, O. Le Fèvre ${ }^{1}$, L. Guzzo ${ }^{2}$, A. Pollo ${ }^{1,2}$, A. Cappi ${ }^{3}$, O. Ilbert ${ }^{4}$, A. Iovino ${ }^{5}$, C. Marinoni ${ }^{5,6}$, \\ H. J. McCracken ${ }^{7,8}$, D. Bottini ${ }^{9}$, B. Garilli ${ }^{9}$, V. Le Brun ${ }^{1}$, D. Maccagni ${ }^{9}$, J. P. Picat ${ }^{10}$, R. Scaramella ${ }^{11}$,
} M. Scodeggio ${ }^{9}$, L. Tresse ${ }^{1}$, G. Vettolani ${ }^{12}$, A. Zanichelli ${ }^{12}$, C. Adami ${ }^{1}$, S. Arnouts ${ }^{1}$, M. Arnaboldi ${ }^{13}$, S. Bardelli ${ }^{3}$, M. Bolzonella ${ }^{4}$, S. Charlot ${ }^{7,14}$, P. Ciliegi ${ }^{3}$, T. Contini ${ }^{10}$, S. Foucaud ${ }^{15}$, P. Franzetti ${ }^{9}$, I. Gavignaud ${ }^{10,16}$, B. Marano ${ }^{4}$,

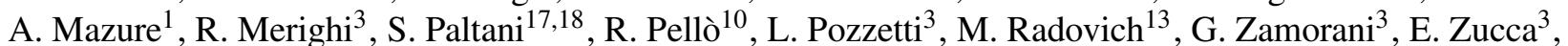
M. Bondi ${ }^{12}$, A. Bongiorno ${ }^{4}$, G. Busarello ${ }^{13}$, O. Cucciati $^{2,14}$, L. Gregorini ${ }^{12}$, F. Lamareille $^{10}$, G. Mathez $^{10}$, Y. Mellier ${ }^{7,8}$, P. Merluzzi ${ }^{13}$, V. Ripepi ${ }^{13}$, and D. Rizzo ${ }^{10}$

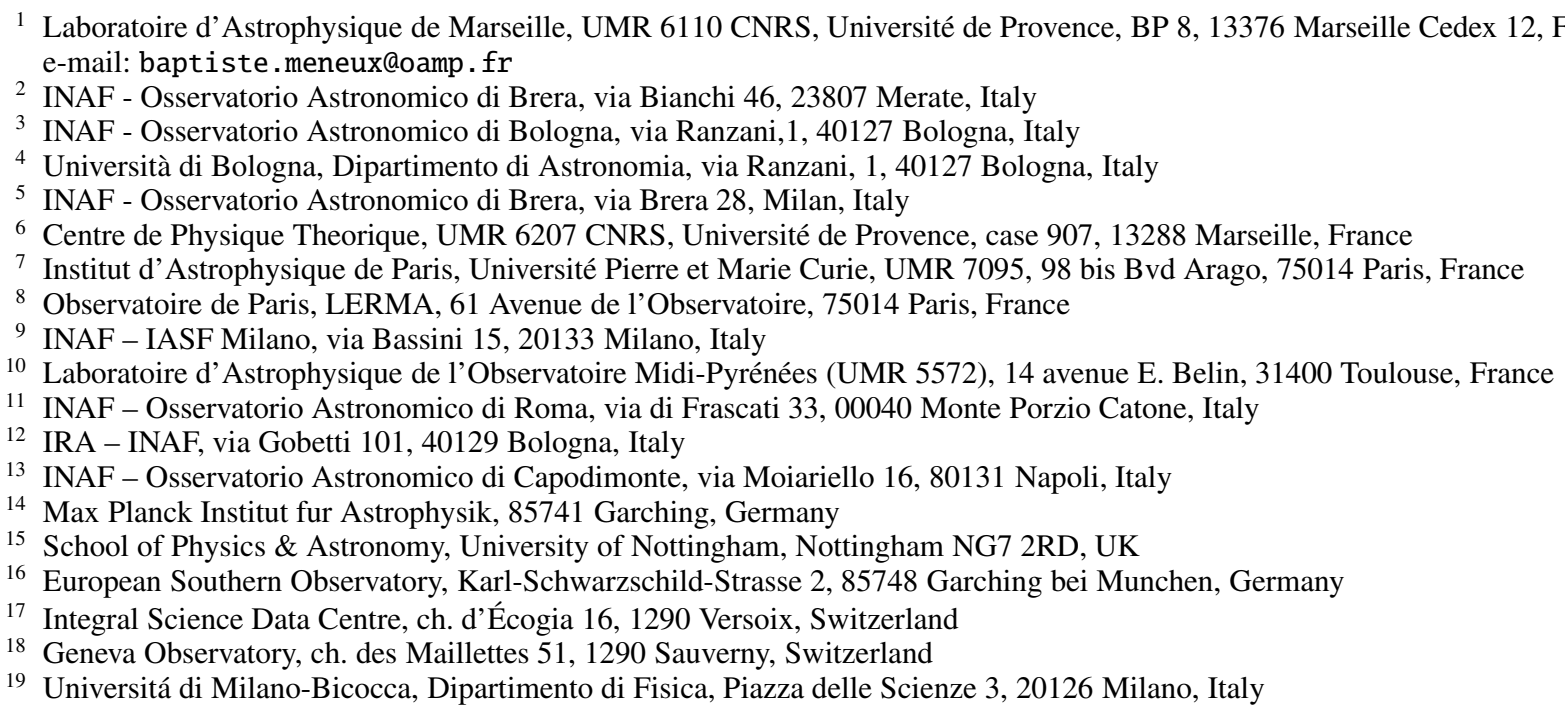

Received 22 November 2005 / Accepted 11 February 2006

\section{ABSTRACT}

\begin{abstract}
We measure the evolution of clustering for galaxies with different spectral types from 6495 galaxies with $17.5 \leq I_{A B} \leq 24$ and measured spectroscopic redshifts in the first epoch VIMOS-VLT Deep Survey (VVDS). We divide our sample into four classes, based on the fit of well-defined galaxy spectral energy distributions on observed multi-color data. We measure the projected correlation function $w_{\mathrm{p}}\left(r_{\mathrm{p}}\right)$ and estimate the best-fit parameters for a power-law real-space correlation function $\xi(r)=\left(r / r_{0}\right)^{-\gamma}$. We find the clustering of early-spectral-type galaxies to be markedly stronger than that of late-type galaxies at all redshifts up to $z \simeq 1.2$. At $z \sim 0.8$, early-type galaxies display a correlation length $r_{0}=4.8 \pm 0.9 h^{-1} \mathrm{Mpc}$, while late types have $r_{0}=2.5 \pm 0.4 h^{-1} \mathrm{Mpc}$. For the latest class of star-forming blue galaxies, we are able to push our clustering measurement to an effective redshift $z \sim 1.4$, for luminous galaxies $\left(M_{B}(A B) \simeq-21\right)$. The clustering of these objects increases up to $r_{0}=3.42 \pm 0.7 h^{-1} \mathrm{Mpc}$ for $z=[1.2,2.0]$. The relative bias between early- and late-type galaxies within our magnitude-limited survey remains approximately constant with $b=1.6 \pm 0.3$ from $z=0$ to $z=1.2$. This result is in agrement with the local findings and fairly robust against different way of classifying red and blue galaxies. When compared to the expected linear growth of mass fluctuations, a natural interpretation of these observations is that: (a) the assembly of massive early type galaxies is already mostly complete in the densest dark matter halos at $z \simeq 1$; (b) luminous late-type galaxies are located in higher-density, more clustered regions of the Universe at $z \simeq 1.5$ than their local low luminous counterpart, indicating that star formation activity is progressively increasing, going back in time, in the higher-density peaks that today are mostly dominated by old galaxies.
\end{abstract}

Key words. surveys - galaxies: evolution - cosmology: large scale structure of Universe - cosmology: observations

* Based on data obtained with the European Southern Observatory Very Large Telescope, Paranal, Chile, program 070.A-9007(A), and on data obtained at the Canada-France-Hawaii Telescope, operated by the CNRS of France, CNRC in Canada and the University of Hawaii and observations obtained with MegaPrime/MegaCam, a joint project of CFHT and CEA/DAPNIA, at the Canada-France-Hawaii Telescope (CFHT) which is operated by the National Research Council (NRC) of Canada, the Institut National des Science de l'Univers of the Centre 


\section{Introduction}

The measurement of the evolution of the clustering of galaxies is one the key elements to understand the evolution of the Universe and the formation of the large-scale structures of the Universe. In the current paradigm, the structures we observe today in the large-scale distribution of galaxies are the result of the gravitational amplification of primordial overdensities in the dark matter density field. Smaller scales are supposed to collapse first, with virialized structures as galaxies, groups and clusters subsequently built up via multiple merging, at a rate of growth essentially governed by the value of the mean matter density $\Omega_{\mathrm{M}}$. In this hierarchical formation scenario, the evolution of the clustering of dark matter halos is now well understood both analytically (Mo \& White 1996; Sheth \& Tormen 1999) and from $N$-body simulations (Jenkins et al. 1998; Kauffmann et al. 1999a; Springel et al. 2005).

The visible component of galaxies is expected to assemble via the collapse of baryonic matter within the dark matter halos. A naive picture would let to conclude that galaxies follow the underlying dark matter density field. The formation and evolution of galaxies is, however, driven by physical processes such as cooling, star formation and feedback, which are not easily linked to the underlying dark matter halos (Kauffmann et al. 1999a). Rather, a general prediction of most current models is that galaxy formation is favored in higher-density regions. Objects forming in regions of larger-than-average density collapse first and, looking back in time, are found to inhabit higher and higher density peaks (i.e., they are more biased). Recent direct measurements of the evolution of galaxy bias up to a redshift $z \sim 1.5$ (Marinoni et al. 2005) provide supporting evidence to this biased picture of galaxy formation.

Although being well known since more that 25 years, the origin of the relationship between galaxy morphological (or almost equivalently, spectral) type and local structure is still not fully understood (e.g. Dressler 1980; Smith et al. 2005; Postman et al. 2005). Whether the higher fraction of early-type galaxies in high- density regions is established ab initio (the so-called nature hypothesis), or rather produced by environmental effects during the life of the galaxy (nurture) is still debated. A practical effect of the morphology-density relation is that early- and late-type galaxies trace differently the density field, with the former class being more clustered (i.e. more biased) than the latter. In addition, more luminous galaxies (which are preferentially earlytypes) are more clustered than less luminous ones, with the two parameters - luminosity and spectral type - playing together in a subtle way (Iovino et al. 1993; Madgwick et al. 2003; Norberg et al. 2002; Zehavi et al. 2002; Loveday Tresse \& Maddox 1999; Guzzo et al. 1997; Benoist et al. 1996). Since the amount of biasing should be related to the mass and formation history of a galaxy, one therefore expects the evolution of the clustering of galaxies with different morphological types to contain important clues on the assembly of the baryonic mass and the origin of galaxy types.

Large deep surveys consistently show that the clustering of the overall galaxy population evolves slowly with time (Coil et al. 2004; Le Fèvre et al. 2005b). Measurements from the VIMOS-VLT Deep Survey (VVDS, Le Fèvre et al. 2005b) indicate that the clustering amplitude of $L^{\star}$ galaxies increases by

National de la Recherche Scientifique (CNRS) of France, and the University of Hawaii. This work is based in part on data products produced at TERAPIX and the Canadian Astronomy Data Centre as part of the Canada-France-Hawaii Telescope Legacy Survey, a collaborative project of NRC and CNRS. a factor $\sim 2.4$ from $z=1.5$ to the present epoch. However, little is known about the clustering evolution of different galaxy types above $z \sim 0.3$. In the CFRS sample, no significant difference was observed between the clustering of red and blue galaxies up to $z \sim 1$ (Le Fèvre et al. 1996), although the sample was too small to reach firm conclusions. Red galaxies at $z \simeq 0.6$ in the CNOC2 survey were found to have a clustering amplitude $\sim 5$ times higher than blue galaxies (Carlberg et al. 1997). Results from the CADIS survey using photometric redshifts indicate that up to $z \sim 1$ early spectral type galaxies are more clustered, with a correlation amplitude 2.1 times larger than late type at $z=$ [0.75-1.07] (Phleps \& Meisenheimer 2003). A more recent and accurate estimate at $\langle z\rangle=0.6$ (Phleps et al. 2005) confirms the same trend among red and blue galaxies with $r_{0}^{\text {red }}=5.39_{-0.28}^{+0.30} h^{-1} \mathrm{Mpc}$ and $r_{0}^{\text {blue }}=3.64_{-0.24}^{+0.25} h^{-1} \mathrm{Mpc}$. At even higher redshifts, the measurement of type is substituted by the selection of specific sub-populations, e.g. Daddi et al. (2003) measure a strong clustering of extremely red $(J-K)$ galaxies at $2 \leq z \leq 4$, arguing that these may represent the progenitors of massive elliptical galaxies at lower redshifts.

In this paper we make an attempt to quantify accurately the contribution of different galaxy types to the observed evolution of clustering using the VVDS first epoch survey. The accuracy of spectroscopic redshifts is used to project the correlation function $\xi\left(r_{\mathrm{p}}, \pi\right)$ in narrow velocity intervals and to measure the correlation length $r_{0}$ and the slope $\gamma$ of the real space correlation function as a function of redshift up to $z \sim 1.2$.

We describe the sample and our galaxy classification in Sect. 2, and the method used to compute the real space correlation function in Sect. 3. The clustering measurements are presented in Sect. 4, and then discussed in Sect. 5. We use a cosmology with $\Omega_{\mathrm{M}}=0.3, \Omega_{\Lambda}=0.7$, throughout the paper. All distances, given in comoving units, and absolute magnitudes are computed with $h=1$ (which denotes $H_{0} / 100 \mathrm{~km} \mathrm{~s}^{-1} \mathrm{Mpc}^{-1}$ ).

\section{VVDS first epoch data}

\subsection{The sample}

We are using the VIMOS-VLT Deep Survey first epoch data described in Le Fèvre et al. (2005a). We concentrate on the VVDS-02h field, and we keep all galaxies with the best redshifts available, with quality flags 2 to 9 (confidence level greater than $80 \%$ in the redshift measurement). AGN are excluded from the sample. The analyzed sample in this paper includes 6495 galaxies with spectroscopic redshifts in an area of $1750 \mathrm{arcmin}^{2}$. The accuracy of the redshift measurement is $\sim 275 \mathrm{~km} \mathrm{~s}^{-1}$. A detailed description of the sample is given in Le Fèvre et al. (2005a).

\subsection{Galaxy classification}

We used the galaxy classification introduced by Zucca et al. (2005) to study the evolution of the luminosity function per galaxy type from the first epoch VVDS data. The classification has been obtained from the $B$ to $I$ band multi-wavelength dataset available in the VVDS-02h field (Le Fèvre et al. 2004a; McCracken et al. 2003), and the spectroscopic redshifts (Le Fèvre et al. 2005a). The $U$ band, which does not cover all the field (Radovich et al. 2003) was not used in order to get a homogeneous classification on the sky. Rest-frame colors have been matched to the empirical set of spectral energy distribution (SED) templates described in Arnouts et al. (1999), made of four observed spectra (Coleman Wu \& Weedman 1980, CWW 
Table 1. The four VVDS galaxy types.

\begin{tabular}{cccc}
\hline \hline Type & & $\begin{array}{c}\text { Number of } \\
\text { objects }\end{array}$ & $\begin{array}{c}\text { Indicated } \\
\text { rest-frame color }\end{array}$ \\
\hline 1 & E/S0 & 645 & $1.3<\left(B_{A B}-I_{A B}\right)$ \\
2 & early spiral & 1004 & $0.95<\left(B_{A B}-I_{A B}\right)<1.3$ \\
3 & late spiral & 2104 & $0.68<\left(B_{A B}-I_{A B}\right)<0.95$ \\
4 & irregular & 2742 & $\left(B_{A B}-I_{A B}\right)<0.68$ \\
& starburst & & \\
\hline
\end{tabular}

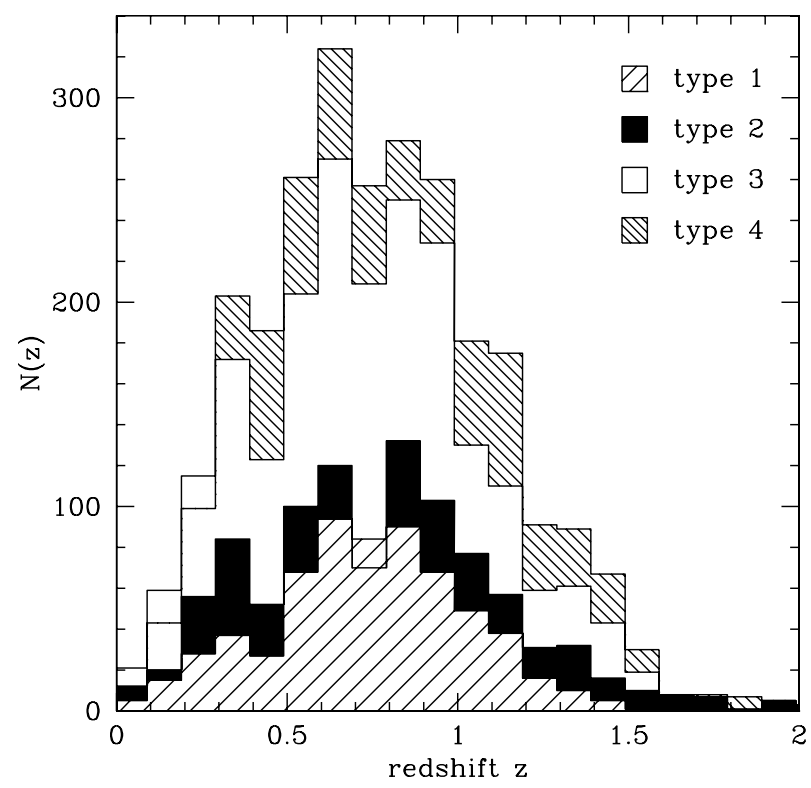

Fig. 1. Redshift distribution of the four galaxy types.

hereafter) and two starburst SEDs computed with GISSEL (Bruzual \& Charlot 1993).

The original spectroscopic sample of 6495 galaxies was divided into four spectral classes from early-type (type 1) to irregular/star-forming galaxies (type 4) based on the CWW templates. The latest type also includes galaxies with SEDs well represented by starburst templates. The properties of these four classes of galaxies are summarized in Table 1 and their redshift distributions are shown in Fig. 1. As a result of the VVDS I-band selection, we are increasingly loosing red early-type galaxies for redshifts $z \gtrsim 1.2$ (Fig. 2) because galaxies are selected from their rest frame UV flux at these redshifts, but late type galaxies continue to be identified up to the highest redshifts discussed in this paper.

As described in Zucca et al. (2005), the evolution of the global luminosity function in the VVDS is strongly driven by type 4 galaxies, which evolve by $\simeq-2$ mag from $z \sim 1.5$ and present a steep faint end luminosity function slope. Conversely, the luminosity function of early type galaxies remains stable over this redshift range. The relative distribution of absolute magnitudes per type presented in Fig. 3 is the result of this evolution.

In summary, the type classification used in this paper is a spectral classification based on a set of spectral templates covering from early type galaxies to late and star-forming galaxies. Although it is tempting to associate spectral type to morphological type, and a close relationship exists between them, the spectral classification used in this paper is more closely related to a color classification from "red" to "blue" galaxies.

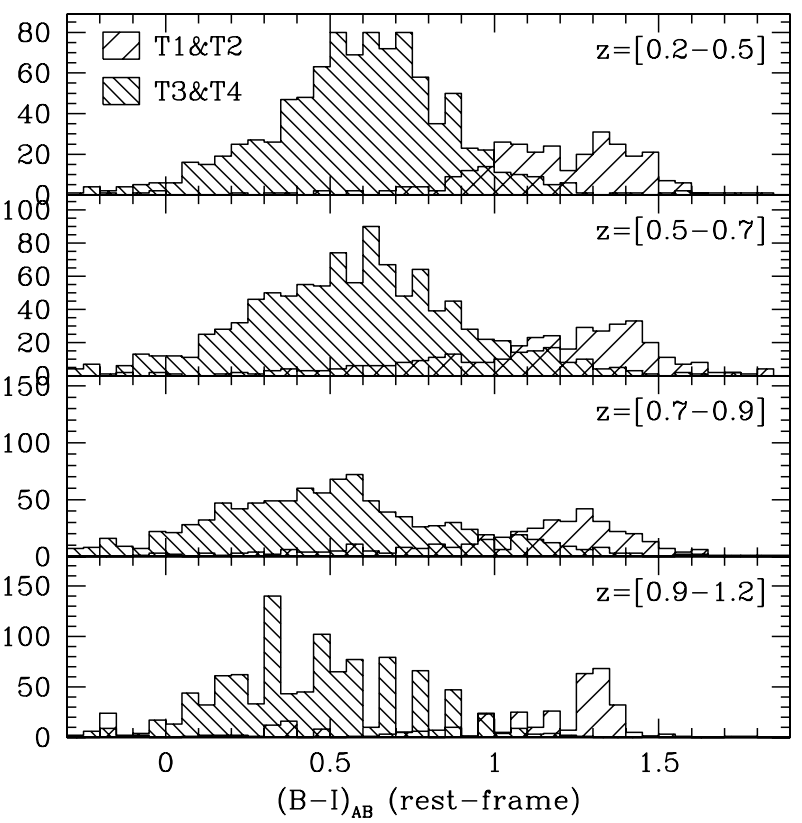

Fig. 2. Rest-frame color $(B-I)_{A B}$ for early-type galaxies (types 1 and 2) and late-type galaxies (type 3 and 4) computed from the best fit of our multi-wavelength dataset with PEGASE. 2 templates (Fioc \& Rocca-Volmerange 1997).

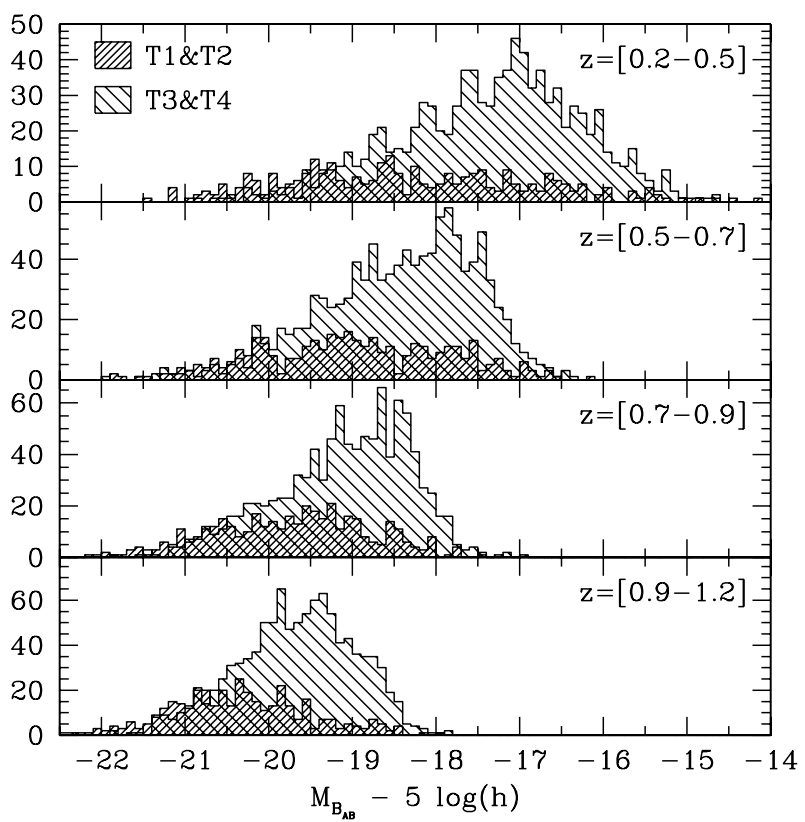

Fig. 3. $B$-band luminosity distribution $M_{B}(A B)$ for early-type galaxies (types 1 and 2) and late-type galaxies (types 3 and 4).

\section{Measurement and associated errors: method}

The method to recover the correlation length $r_{0}$ and the slope $\gamma$ of the real-space correlation function is well detailed in the paper of Pollo et al. (2005). We are focusing here on the most critical aspects of the method when applied to compute the clustering properties as a function of galaxy type.

\subsection{The real space correlation functions parameters}

We computed the bi-dimensional two-point correlation function $\xi\left(r_{\mathrm{p}}, \pi\right)$ for each galaxy type, in different redshift bins chosen to 
maximize the number of objects hence the signal to noise ratio of the clustering measurement. This was estimated using the Landy \& Szalay (1993) estimator

$\xi\left(r_{\mathrm{p}}, \pi\right)=\frac{N_{\mathrm{R}}\left(N_{\mathrm{R}}-1\right)}{N_{\mathrm{G}}\left(N_{\mathrm{G}}-1\right)} \frac{G G\left(r_{\mathrm{p}}, \pi\right)}{R R\left(r_{\mathrm{p}}, \pi\right)}-\frac{N_{\mathrm{R}}-1}{N_{\mathrm{G}}} \frac{G R\left(r_{\mathrm{p}}, \pi\right)}{R R\left(r_{\mathrm{p}}, \pi\right)}+1$

where $N_{\mathrm{G}}$ is the mean galaxy density (or, equivalently, the total number of objects) in the survey; $N_{\mathrm{R}}$ is the mean density of a catalog of random points distributed within the same survey volume; $G G\left(r_{\mathrm{p}}, \pi\right)$ is the number of independent galaxy-galaxy pairs with separation between $r_{\mathrm{p}}$ and $r_{\mathrm{p}}+\mathrm{d} r_{\mathrm{p}}$ perpendicular to the line-of-sight and between $\pi$ and $\pi+\mathrm{d} \pi$ along the line of sight; $R R\left(r_{\mathrm{p}}, \pi\right)$ is the number of independent random-random pairs within the same interval of separations and $G R\left(r_{\mathrm{p}}, \pi\right)$ represents the number of galaxy-random cross pairs.

The real-space correlation function $\xi(r)$ represents the excess probability for a given pair of galaxies to be observed at a separation $r$ (Peebles 1980). It can be derived from $\xi\left(r_{\mathrm{p}}, \pi\right)$ using the formalism of Davis \& Peebles (1983), computing $w_{\mathrm{p}}\left(r_{\mathrm{p}}\right)$, the projection of $\xi\left(r_{\mathrm{p}}, \pi\right)$ along the line of sight.

$w_{\mathrm{p}}\left(r_{\mathrm{p}}\right) \equiv 2 \int_{0}^{\infty} \xi\left(r_{\mathrm{p}}, \pi\right) \mathrm{d} y=2 \int_{0}^{\infty} \xi\left[\left(r_{\mathrm{p}}^{2}+y^{2}\right)^{1 / 2}\right] \mathrm{d} y$

In practice, the upper integration limit must be chosen finite, as to include the real signal, without adding extra noise which is dominant above a certain $\pi$. Based on direct tests with the GalICS mock samples (Blaizot et al. 2005), we choose a value $\pi_{\max }=20 \mathrm{~h}^{-1} \mathrm{Mpc}$. This is high enough to sum the real signal, without adding too much noise. It is compatible with the VVDS velocity measurement error of $\sim 275 \mathrm{~km} \mathrm{~s}^{-1}$. Assuming a powerlaw form for the real-space correlation function $\xi(r)=\left(r / r_{0}\right)^{\gamma}$, the integral in Eq. (2) can be solved analytically in terms of Gamma functions, yielding a $w_{\mathrm{p}}\left(r_{\mathrm{p}}\right)$ which is itself a power law. The fitting method of the measured $w_{\mathrm{p}}\left(r_{\mathrm{p}}\right)$, detailed in Pollo et al. (2005), provides the values of the correlation length $r_{0}$ and the slope $\gamma$ with their associated errors. A specific treatment have been applied to compute the clustering properties per galaxy types, as described in Sects. 3.2 and 3.3.

\subsection{Biases and weights for each spectral class}

In order to correct for the observational biases introduced by the VVDS observing strategy in the VVDS-02h field, we used the same correction scheme explained in Pollo et al. (2005). The main biases are the incompleteness in redshift measurement and the complex geometry of the field. This results in a spatial sampling that changes as a function of position in the field. To account for galaxies with unknown redshift (either unobserved or discarded because of a low redshift quality flag), the observed galaxies are given a weight. The goal of this weight is to recover the true number of galaxy pairs at a given angular scale.

The computation of this local weight takes into account the local number of galaxies (with and without a redshift) around a given galaxy in the spectroscopic sample. The parent photometric sample used for this computation must have the same properties as the spectroscopic one. For instance, the weight computed for the early type galaxies from the spectroscopic sample must make a reference only to the early type galaxies in the underlying complete photometric sample, without including other galaxy types. In this approach, the parent photometric catalogue used to compute the weight must therefore only contain galaxies with the same properties as those of the spectroscopic sample.
Photometric redshifts of all the galaxies in the VVDS-02h have been derived from $B V R I$ photometry (Le Fèvre et al. 2004a; McCracken et al. 2003), and ugriz bands from the CFHT Legacy Survey. Details are presented in Ilbert et al. (in prep.). The photometric redshifts and rest-frame magnitudes were then used to give a spectral type to all these galaxies, according to the method explained in Sect. 2.2. The complete parent photometric catalogue was then divided in four catalogues, one for each type. Note that the photometric redshifts are only used to define a spectral type to each galaxy in the whole photometric cata$\log$ in order to compute the weights associated to each galaxy type, in addition to the spectroscopically measured galaxies. Photometric redshifts are not directly used here in the computation of the correlation function.

\subsection{Error measurement}

Even if our spectroscopic sample is the largest to date at this depth, and despite the angular size of the VVDS-02h $\left(1750 \operatorname{arcmin}^{2}\right)$, the uncertainties associated to the measurement of the power-law parameters of the real-space correlation function $\xi(r)$ are largely dominated by the cosmic variance.

To assess this, we have constructed 50 VVDS-02h mock surveys from the GalICS simulation (Blaizot et al. 2005). GalICS is a semi-analytic model for galaxy evolution post-processed to a large cosmological $N$-body simulation. These mock surveys are quasi-independent and are built applying all the VVDS-02h observing biases: the complex geometry of the field, the spectroscopic selection function, the redshift measurement accuracy, the incompleteness (see Pollo et al. 2005, for more information). We use the clustering variance in the mock samples to quantify the cosmic variance expected on the correlation length $r_{0}$ and the slope $\gamma$ of the real-space correlation function.

Our method to assign a spectral type to a given galaxy does not work so well with the GalICS simulations. We were not able to recover a realistic redshift distribution for the four spectral types using the CFH12k BVRI apparent magnitudes produced by GalICS. For example, there were no type 1 (elliptical) galaxies beyond $z \sim 0.5$ in most of the 50 GalICS cones. Conversely, the galaxy type classification given in for GalICS data do not match ours. This "Morphological" type classification is based on rest-frame $B$-band (bulge+burst)-to-disc luminosity ratio. This provides a huge "spiral" class (on average $~ 90 \%$ of the full dataset), and two equivalent (in number of objects) "elliptical" and "lenticular" classes. These two last classes could match our type 1 class. But then, this is quite difficult to divide the huge "spiral" class in our 3 later types.

Computing error measurements from a bootstrap resampling method slightly under-estimates the real variance: if we consider the full VVDS-02h data set (Le Fèvre et al. 2005b), the relative errors computed from GalICS are $\sim 1.5-2$ times greater than error computed from bootstrap resampling. We expect similar factor when dividing our sample per spectral type.

Therefore the approach that has been chosen to compute measurement errors consists in taking randomly, in each of the 50 mock VVDS-02h surveys, a proportion of galaxies equal to that found in the VVDS-02h spectroscopic sample for a given spectral class. Even if the clustering measured is the one of the "full" population with higher incertainties, the statistic of each "type" is representative of the real one. The variance from field to field is artificially increased by a broader range of clustering properties in the simulated data, and therefore, this method is very conservative in the way that it is expected to produce errors for the observed data larger than in reality. 


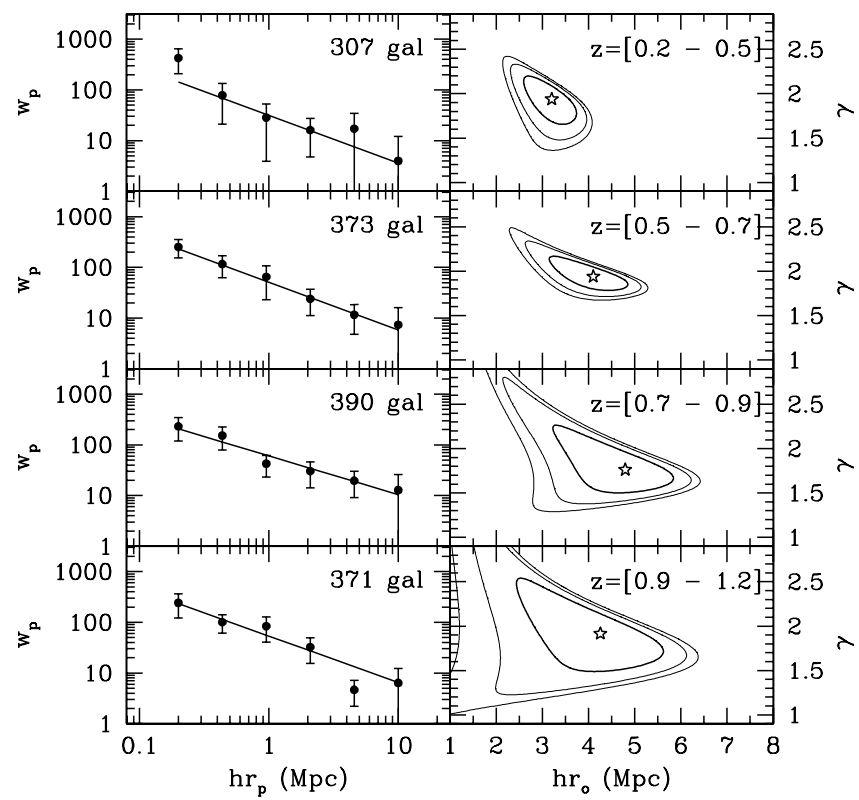

Fig. 4. (Left) Correlation function $w_{\mathrm{p}}\left(r_{\mathrm{p}}\right)$ as a function of redshift for early-type galaxies with types 1 and 2 . (Right) Error contours $(68 \%$, $90 \%$ and $95 \%$ likelihood levels) associated to the measurement of the correlation length $r_{0}$ and slope $\gamma$ of the correlation function.

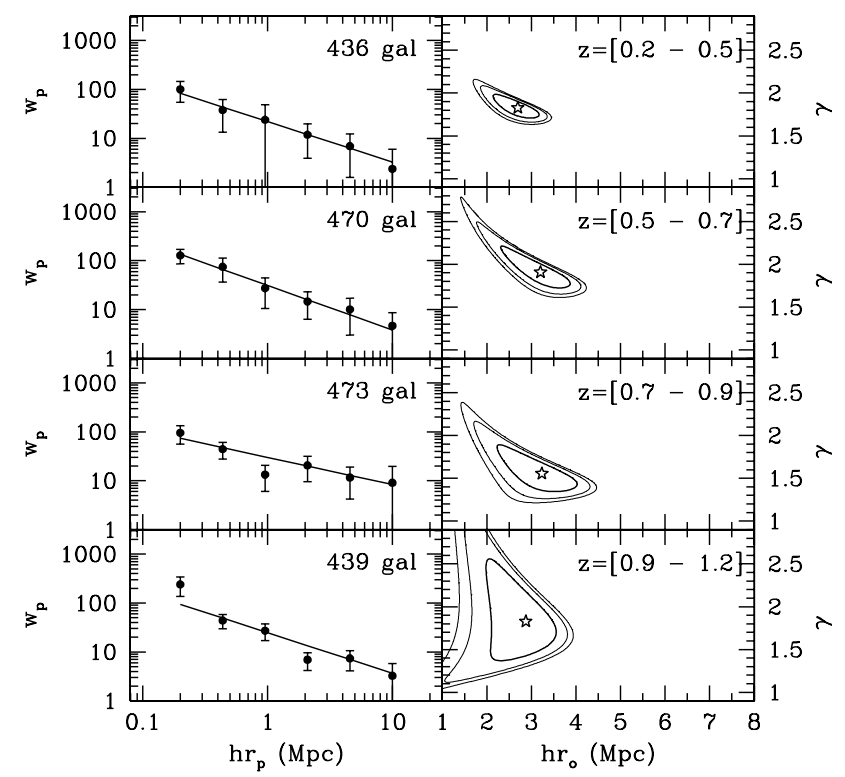

Fig. 5. Same as Fig. 4 for galaxies with type 3 .

\section{Clustering evolution for each galaxy type}

\subsection{Measurement of $r_{0}$ and $\gamma$}

We have computed the correlation function $\xi\left(r_{\mathrm{p}}, \pi\right)$ and its projection $w_{\mathrm{p}}\left(r_{\mathrm{p}}\right)$ in the VVDS-02h field, for each galaxy type of our classification, in increasing redshift slices. In order to improve the clustering signal, and depending on the redshift bin considered, we merged the two earliest-type galaxy samples $T 1$ and $T 2$ and the two latest types $T 3$ and $T 4$. Merging types 1 and 2 would clearly dilute the clustering signal of type 1 galaxies if they are more strongly clustered than type 2 . The measurements of $w_{\mathrm{p}}\left(r_{\mathrm{p}}\right)$ are presented in Figs. 4 to 7.

The projection $w_{\mathrm{p}}\left(r_{\mathrm{p}}\right)$ has been fitted by a power-law on scales $0.1 \leq r_{\mathrm{p}} \leq 10 \mathrm{~h}^{-1} \mathrm{Mpc}$. The values of the correlation

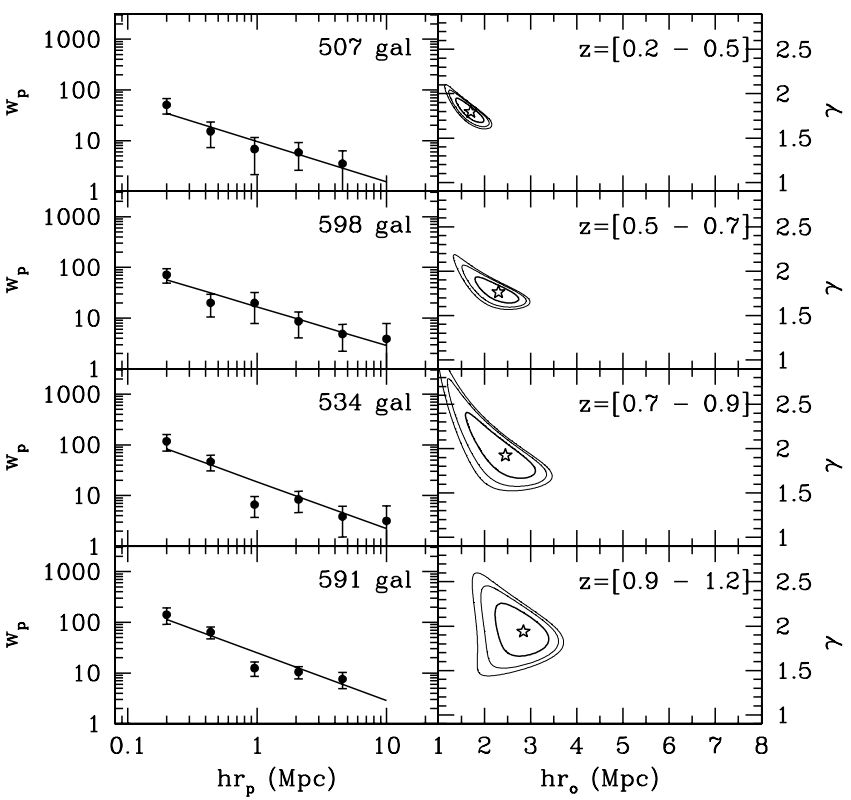

Fig. 6. Same as Fig. 4 for galaxies with type 4 .

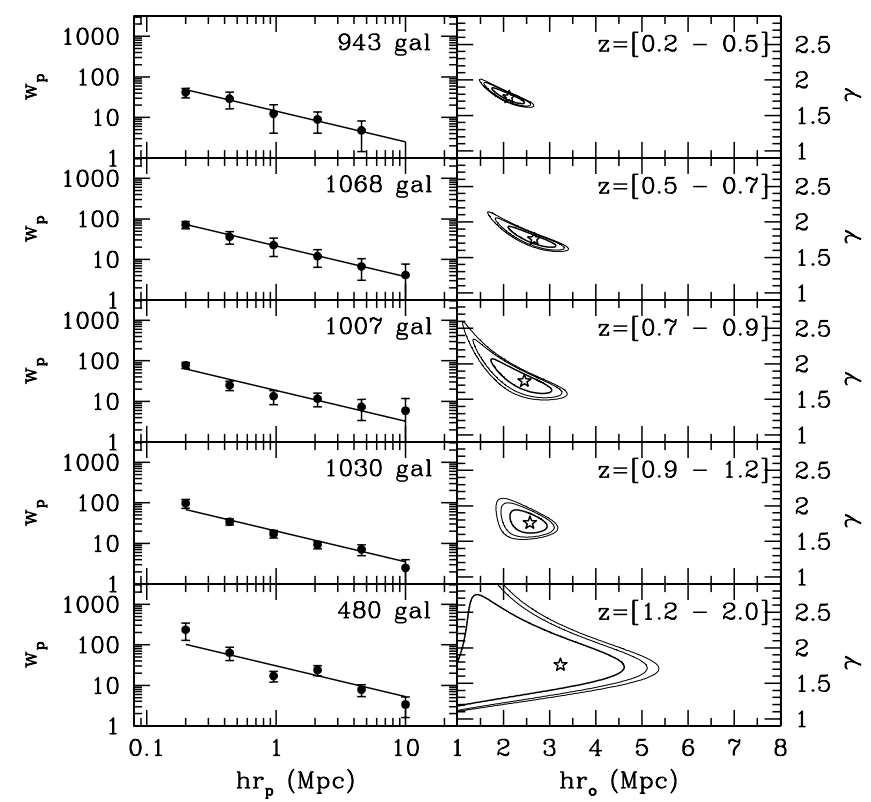

Fig. 7. Same as Fig. 4 for galaxies with types 3 and 4.

length $r_{0}$ and the slope $\gamma$ of the real-space correlation function are summarized in Table 2. A clear difference in clustering strength is observed between the 3 classes of galaxies: T1\&2, $\mathrm{T} 3$ and T4. We find that on average the clustering strength of early type galaxies is 1.8 times larger than the clustering strength of type 4 galaxies. This is observed over the full redshift range $0.2 \leq z \leq 1.2$ and this result is significant. The probability that the correlation lengths of these two populations are the same is indeed only $2.3 \%$ for the redshift bin $z=[0.2,0.5], 2.7 \%$ for $z=[0.5,0.7], 2.2 \%$ for $z=[0.7,0.9]$, and $14.5 \%$ for $z=[0.9,1.2]$.

The evolution of the clustering length for the different classes of galaxies is presented in Fig. 8. An overall increase in the clustering of all galaxy types is observed up to $z \simeq 1$. In the range $z=[0.9,1.2]$, there is a slight indication for a weakening in the clustering of early type galaxies. The clustering length of late type / star-forming galaxies continues to rise up to redshift $\sim 2$. 
Table 2. Measurement of the clustering length for galaxy subsamples ranging from $z=0.2$ to $z=2$, letting the slope $\gamma$ free or fixing the slope to the average value measured for a given type. The absolute $M_{B_{A B}}$ magnitude and number of objects are indicated.

\begin{tabular}{|c|c|c|c|c|c|c|c|c|}
\hline Type & $\begin{array}{c}\text { Number of } \\
\text { galaxies }\end{array}$ & $\begin{array}{c}\text { Redshift } \\
\text { range }\end{array}$ & $\begin{array}{c}\text { Effective } \\
\text { redshift }\end{array}$ & $\begin{array}{c}M_{B_{A B}} \\
\text { mean }\end{array}$ & $\begin{array}{l}5 \log (h) \\
\text { median } \\
\end{array}$ & $\begin{array}{c}r_{0}(z) \\
\left(h^{-1} \mathrm{Mpc}\right) \\
\end{array}$ & $\gamma$ & $\begin{array}{l}r_{0}(z) \text { at fixed } \gamma \\
\left(h^{-1} \mathrm{Mpc}\right)\end{array}$ \\
\hline 1 & $\begin{array}{l}164 \\
332 \\
237 \\
\end{array}$ & $\begin{array}{l}{[0.2-0.6]} \\
{[0.6-1.0]} \\
{[0.8-1.2]} \\
\end{array}$ & $\begin{array}{l}0.427 \\
0.779 \\
0.964 \\
\end{array}$ & $\begin{array}{l}-18.799 \\
-19.755 \\
-20.324 \\
\end{array}$ & $\begin{array}{l}-19.020 \\
-19.819 \\
-20.347 \\
\end{array}$ & $\begin{array}{l}3.14_{-0.94}^{+0.76} \\
4.35_{-0.98}^{+0.68} \\
3.89_{-1.44}^{+1.04} \\
\end{array}$ & $\begin{array}{l}2.41_{-0.37}^{+0.33} \\
2.01_{-0.29}^{+0.46} \\
2.04_{-0.45}^{+0.80} \\
\end{array}$ & $\begin{array}{l}3.51_{-0.71}^{+0.65}(\gamma=2.15) \\
3.93_{-0.74}^{+0.55} \\
3.62_{-1.26}^{+0.89}\end{array}$ \\
\hline 2 & $\begin{array}{l}299 \\
420 \\
358 \\
\end{array}$ & $\begin{array}{l}{[0.2-0.6]} \\
{[0.6-1.0]} \\
{[0.8-1.2]} \\
\end{array}$ & $\begin{array}{l}0.409 \\
0.796 \\
0.972 \\
\end{array}$ & $\begin{array}{l}-18.352 \\
-19.525 \\
-20.059 \\
\end{array}$ & $\begin{array}{l}-18.419 \\
-19.503 \\
-20.115 \\
\end{array}$ & $\begin{array}{l}2.79_{-0.44}^{+0.42} \\
4.92_{-0.77}^{+0.72} \\
4.35_{-1.14}^{+0.96} \\
\end{array}$ & $\begin{array}{l}1.88_{-0.14}^{+0.17} \\
1.82_{-0.20}^{+0.27} \\
2.00_{-0.26}^{+0.38} \\
\end{array}$ & $\begin{array}{l}2.76_{-0.32}^{+0.35}(\gamma=1.90) \\
4.89_{-0.58}^{+0.58} \\
4.62_{-0.96}^{+0.85}\end{array}$ \\
\hline 3 & $\begin{array}{l}437 \\
470 \\
473 \\
439 \\
191 \\
\end{array}$ & $\begin{array}{l}{[0.2-0.5]} \\
{[0.5-0.7]} \\
{[0.7-0.9]} \\
{[0.9-1.2]} \\
{[1.2-2.0]} \\
\end{array}$ & $\begin{array}{l}0.346 \\
0.606 \\
0.810 \\
1.027 \\
1.394 \\
\end{array}$ & $\begin{array}{l}-17.478 \\
-18.665 \\
-19.296 \\
-19.843 \\
-20.756 \\
\end{array}$ & $\begin{array}{l}-17.332 \\
-18.601 \\
-19.212 \\
-19.835 \\
-20.751 \\
\end{array}$ & $\begin{array}{l}2.69_{-0.54}^{+0.50} \\
3.21_{-0.60}^{+0.69} \\
3.24_{-0.65}^{+0.62} \\
2.88_{-0.44}^{+0.44} \\
3.60_{-1.38}^{+0.83} \\
\end{array}$ & $\begin{array}{l}1.83_{-0.11}^{+0.16} \\
1.91_{-0.24}^{+0.24} \\
1.56_{-0.15}^{+0.22} \\
1.83_{-0.27}^{+0.36} \\
1.73_{-0.46}^{+0.35} \\
\end{array}$ & $\begin{array}{l}2.73_{-0.42}^{+0.44}(\gamma=1.78) \\
3.33_{-0.59}^{+0.54} \\
3.21_{-0.60}^{+0.60} \\
2.82_{-0.50}^{+0.49} \\
3.65_{-3.04}^{+1.02}\end{array}$ \\
\hline 4 & $\begin{array}{l}507 \\
598 \\
534 \\
591 \\
289 \\
\end{array}$ & $\begin{array}{l}{[0.2-0.5]} \\
{[0.5-0.7]} \\
{[0.7-0.9]} \\
{[0.9-1.2]} \\
{[1.2-2.0]} \\
\end{array}$ & $\begin{array}{l}0.366 \\
0.604 \\
0.807 \\
1.036 \\
1.394 \\
\end{array}$ & $\begin{array}{l}-17.255 \\
-18.277 \\
-18.944 \\
-19.553 \\
-20.441 \\
\end{array}$ & $\begin{array}{l}-17.170 \\
-18.136 \\
-18.829 \\
-19.494 \\
-20.397 \\
\end{array}$ & $\begin{array}{l}1.71_{-0.31}^{+0.30} \\
2.31_{-0.40}^{+0.40} \\
2.46_{-0.57}^{+0.49} \\
2.85_{-0.31}^{+0.36} \\
3.30_{-0.87}^{+0.64} \\
\end{array}$ & $\begin{array}{l}1.80_{-0.12}^{+0.16} \\
1.76_{-0.10}^{+0.14} \\
1.92_{-0.19}^{+0.31} \\
1.94_{-0.15}^{+0.18} \\
1.92_{-0.32}^{+0.36} \\
\end{array}$ & $\begin{array}{l}1.71_{-0.30}^{+0.30}(\gamma=1.86) \\
2.28_{-0.39}^{+0.42} \\
2.49_{-0.58}^{+0.53} \\
2.91_{-0.37}^{+0.40} \\
3.27_{-2.35}^{+1.01}\end{array}$ \\
\hline $1 \& 2$ & $\begin{array}{l}307 \\
373 \\
390 \\
371 \\
\end{array}$ & $\begin{array}{l}{[0.2-0.5]} \\
{[0.5-0.7]} \\
{[0.7-0.9]} \\
{[0.9-1.2]} \\
\end{array}$ & $\begin{array}{l}0.346 \\
0.604 \\
0.814 \\
1.032 \\
\end{array}$ & $\begin{array}{l}-18.327 \\
-18.996 \\
-19.719 \\
-20.333 \\
\end{array}$ & $\begin{array}{l}-18.512 \\
-19.020 \\
-19.635 \\
-20.383 \\
\end{array}$ & $\begin{array}{l}3.21_{-0.60}^{+0.58} \\
4.11_{-0.76}^{+0.66} \\
4.80_{-0.93}^{+0.81} \\
4.26_{-0.95}^{+0.87} \\
\end{array}$ & $\begin{array}{l}1.94_{-0.29}^{+0.27} \\
1.94_{-0.12}^{+0.19} \\
1.76_{-0.18}^{+0.30} \\
1.92_{-0.24}^{+0.31} \\
\end{array}$ & $\begin{array}{l}3.21_{-0.40}^{+0.40}(\gamma=1.89) \\
4.14_{-0.53}^{+0.53} \\
4.41_{-0.69}^{+0.62} \\
4.32_{-0.90}^{+0.79}\end{array}$ \\
\hline $3 \& 4$ & $\begin{array}{c}943 \\
1068 \\
1007 \\
1030 \\
480 \\
\end{array}$ & $\begin{array}{l}{[0.2-0.5]} \\
{[0.5-0.7]} \\
{[0.7-0.9]} \\
{[0.9-1.2]} \\
{[1.2-2.0]}\end{array}$ & $\begin{array}{l}0.357 \\
0.605 \\
0.809 \\
1.032 \\
1.394 \\
\end{array}$ & $\begin{array}{l}-17.358 \\
-18.448 \\
-19.109 \\
-19.677 \\
-20.566 \\
\end{array}$ & $\begin{array}{l}-17.230 \\
-18.321 \\
-19.008 \\
-19.626 \\
-20.510 \\
\end{array}$ & $\begin{array}{l}2.12_{-0.36}^{+0.35} \\
2.66_{-0.45}^{+0.43} \\
2.49_{-0.42}^{+0.42} \\
2.58_{-0.22}^{+0.25} \\
3.42_{-0.73}^{+0.66} \\
\end{array}$ & $\begin{array}{l}1.78_{-0.09}^{+0.14} \\
1.74_{-0.09}^{+0.15} \\
1.67_{-0.11}^{+0.17} \\
1.86_{-0.08}^{+0.11} \\
1.99_{-0.20}^{+0.24} \\
\end{array}$ & $\begin{array}{l}2.12_{-0.32}^{+0.32}(\gamma=1.76) \\
2.66_{-0.41}^{+0.41} \\
2.46_{-0.50}^{+0.48} \\
2.58_{-0.25}^{+0.28} \\
3.24_{-1.23}^{+0.90}\end{array}$ \\
\hline
\end{tabular}

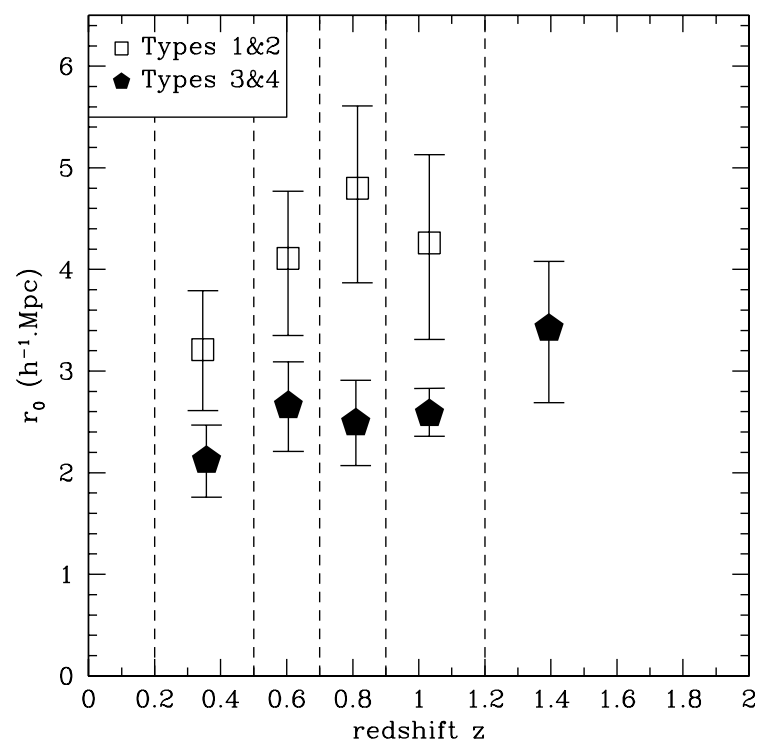

Fig. 8. Correlation length $r_{0}$ as a function of redshift for early (T1\&2) and late $(\mathrm{T} 3 \& 4)$ type galaxies. Here the slope $\gamma$ is a free parameter.

\subsection{Relative bias and its evolution}

Since it is not possible to measure directly the real-space correlation function of the mass, any attempt to measure the bias $b$ from galaxy samples has either to assume the knowledge of the background cosmological parameters (e.g. Marinoni et al. 2005), or to obtain the mass variance from other observables like the Cosmic Microwave Background (e.g. Lahav et al. 2002).
The simplest definition of the bias $b$ that links linearly the mass density field $\rho$ of the mass to the galaxy density field $n$ is (for a fixed scale $R$ over which $\rho$ and $n$ are measured)

$\frac{\delta n}{\langle n\rangle}=b \frac{\delta \rho}{\langle\rho\rangle}$,

where $b$ is simply a number, independent on scale and on the local value of $\delta \rho /\langle\rho\rangle$ hypothesis. Similarly, but in a statistical rather than deterministic way, the bias can be defined in terms of $r m s$ values of the galaxy and density fields

$\left(\frac{\delta n}{\langle n\rangle}\right)_{\mathrm{rms}}=b\left(\frac{\delta \rho}{\langle\rho\rangle}\right)_{\mathrm{rms}}$.

With this definition, $b$ is simply a ratio if the square root of the variance of the galaxy and mass density fields measured over a given scale $R$, i.e. $\sigma_{R}$ (galaxies) $=b \sigma_{R}$ (mass). In our case, we can measure directly the relative bias between two populations $A$ and $B$, as

$\frac{b_{A}}{b_{B}}=\frac{\sigma_{8}^{A}(r)}{\sigma_{8}^{B}(r)}$

where $\sigma_{8}$ can be computed from the measured correlation function as

$\sigma_{R}^{2}=\frac{3}{R^{3}} \int_{0}^{R} r^{2} \xi(r) \mathrm{d} r$,

which, for power-law form of $\xi(r)$, can be written as (Peebles 1980)

$\sigma_{8}=\sqrt{C_{\gamma}\left(\frac{r_{0}}{8 \mathrm{Mpc} / h}\right)^{\gamma}}$, 


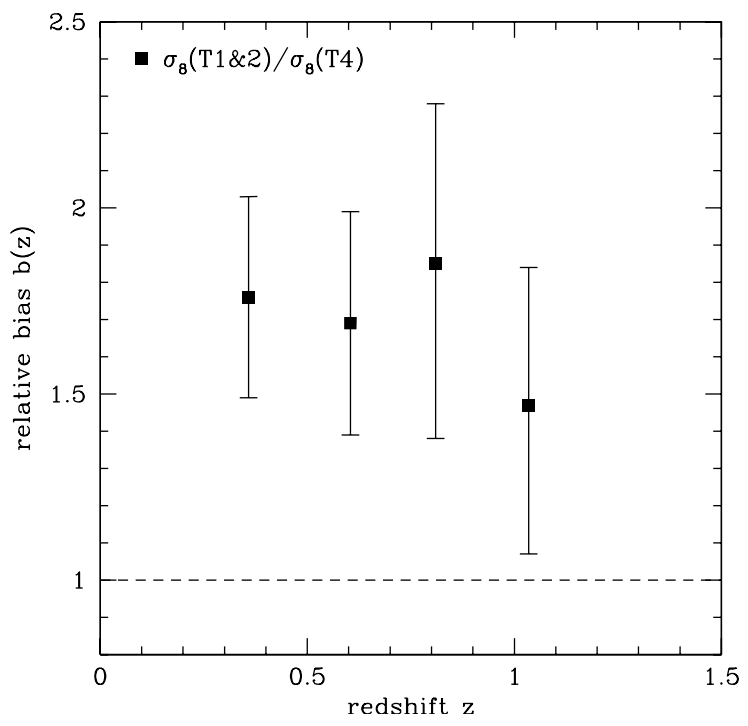

Fig. 9. Relative bias between populations of galaxies for a $8 h^{-1} \mathrm{Mpc}$ scale: $b_{A} / b_{B}=\sigma_{8}^{A} / \sigma_{8}^{B}$.

with

$C_{\gamma}=\frac{72}{(3-\gamma)(4-\gamma)(6-\gamma) 2^{\gamma}}$.

Figure 9 shows the relative bias between the populations $\mathrm{T} 1+\mathrm{T} 2$ and T4 at different redshifts, measured using Eq. (7) in spheres with radius $r=8 h^{-1} \mathrm{Mpc}$. The average value is $b=1.65 \pm 0.3$ consistent with being constant up to the highest redshift investigated. This result seems to be mostly insensitive to the way the two populations are selected. For example we find a relative bias $b=1.6 \pm 0.3$ when the red and blue samples are selected according to color bimodality (see next section). Note, however that by considering the intermediate-type population in our comparison, we find a relative bias $b=1.3 \pm 0.2$ between $\mathrm{T} 1+\mathrm{T} 2$ and T3, which is marginally $(\sim 1 \sigma)$ lower than between $\mathrm{T} 1+\mathrm{T} 2$ and $\mathrm{T} 4$.

This is in agreement with what is found locally for nearly the same color-selected populations. For example Wild et al. (2005) using the 2dFGRS find on the same $8 h^{-1}$ Mpc scale, $b \sim 1.5$. Moreover, at higher redshifts, this result is in agreement with what has been found in a previous VVDS study (Marinoni et al. 2005), from the same data but using a different method.

\section{Rest-frame color bimodality}

It has become clear in recent years that the galaxy rest-frame colors show a bimodal distribution (Strateva et al. 2001), i.e. that in a (rest-frame) color-magnitude diagram galaxies tend to segregate between a "red sequence" (similar, but less tight than that observed for cluster galaxies) and a "blue cloud". This behavior seems also to be present at high redshift (Bell et al. 2004; Giallongo et al. 2005). From the SDSS data, Strateva et al. (2001) find that the blue cloud contains mainly late (spiral) morphological types while the bulk of the red sequence consists of bulge-dominated, early-type galaxies (see also Weiner et al. 2005). This provides a natural recipe to split galaxies into two populations of "red" and "blue" objects and there have been already attempts to study the clustering of galaxies separately for the two classes defined in this way at intermediate redshifts (e.g. Phleps et al. 2005).

It is therefore interesting to check how our analysis as a function of SED-defined types compares to a simpler sub-division following the bimodal distribution.

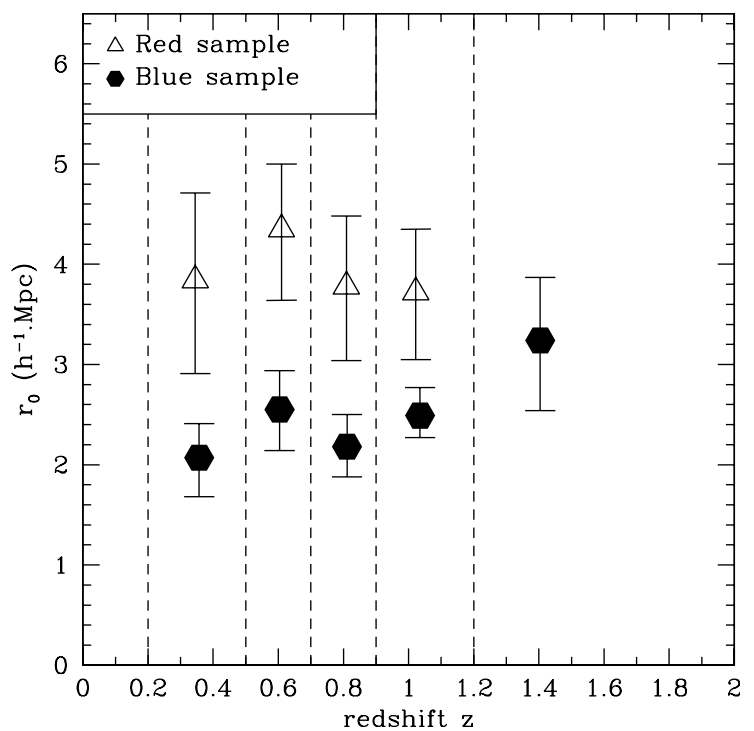

Fig. 10. Correlation length $r_{0}$ as a function of redshift for the red and blue samples. Here the slope $\gamma$ is a free parameter.

Table 3. Relative bias between early-type and late-type galaxies (from spectral type classification), and "red" vs. "blue" galaxies (separated using the color bi-modality).

\begin{tabular}{lcc}
\hline \hline & $\begin{array}{c}\text { Redshift } \\
\text { range }\end{array}$ & $\begin{array}{c}\text { Relative } \\
\text { bias }\end{array}$ \\
\hline T1\&2/T3 & {$[0.2-0.5]$} & $1.18_{-0.22}^{+0.23}$ \\
& {$[0.5-0.7]$} & $1.27_{-0.28}^{+0.26}$ \\
& {$[0.7-0.9]$} & $1.39_{-0.27}^{+0.26}$ \\
& {$[0.9-1.2]$} & $1.45_{-0.37}^{+0.34}$ \\
\hline T1\&2/T4 & {$[0.2-0.5]$} & $1.76_{-0.27}^{+0.27}$ \\
& {$[0.5-0.7]$} & $1.69_{-0.30}^{+0.30}$ \\
& {$[0.7-0.9]$} & $1.85_{-0.47}^{+0.43}$ \\
& {$[0.9-1.2]$} & $1.47_{-0.40}^{+0.37}$ \\
\hline "red"/"blue" & {$[0.2-0.5]$} & $1.69_{-0.47}^{+0.43}$ \\
& {$[0.5-0.7]$} & $1.63_{-0.34}^{+0.32}$ \\
& {$[0.7-0.9]$} & $1.45_{-0.30}^{+0.29}$ \\
& {$[0.9-1.2]$} & $1.45_{-0.27}^{+0.27}$ \\
\hline
\end{tabular}

We have thus split our data into a blue and red sample, following the rest-frame color-magnitude relations suggested by Giallongo et al. (2005) and measured the projected function $w_{\mathrm{p}}\left(r_{\mathrm{p}}\right)$ and the best-fit power-law correlation function $\xi(r)$ in different redshift bins. The resulting evolution of the correlation length is shown in Fig. 10, with the measured values for $r_{0}$ and $\gamma$ summarized in Table 5. The associated errors have been computed in the usual way, constructing blue and red mock catalogues from the GalICS simulations, keeping the same proportion of red (blue) objects as in the real VVDS data.

Similarly to what we found for early-type classes, redsequence galaxies exhibit a larger clustering length $\left(r_{0} \sim\right.$ $4 h^{-1} \mathrm{Mpc}$ ), with little dependence on redshift. Comparison to Fig. 8 indicates that the classification into rest-frame red and blue galaxies is substantially equivalent to our classification into spectral late-types, producing samples with similar clustering evolution properties.

The relative bias between red and blue galaxies is measured to be on average $b=1.6 \pm 0.3$ between $z \sim 0.3$ and $z \sim 1$ (see Table 3), a value comparable with that derived using the spectral type classification. 
Table 4. Number of galaxies and mean $B$ rest-frame absolute magnitude $(h=1)$ of the sample separated using the color bimodality. The number of "red" and "blue" galaxies classified as early (T1\&2) and late (T3\&4) spectral types are identified. We note that the populations of "red" and T1\&2 as well as the populations of "blue" and T3\&4 galaxies are mostly the same, except for a significant cross-over population which could explain the observed difference in clustering (see text).

\begin{tabular}{|c|c|c|c|c|}
\hline \multirow{2}{*}{$\begin{array}{l}\text { Redshift } \\
\text { range }\end{array}$} & \multicolumn{2}{|c|}{ Red } & \multicolumn{2}{|c|}{ Blue } \\
\hline & $\mathrm{T} 1 \& 2$ & $\mathrm{~T} 3 \& 4$ & $\mathrm{~T} 1 \& 2$ & $\mathrm{~T} 3 \& 4$ \\
\hline \multirow[t]{2}{*}{$0.2-0.5$} & 221 & 49 & 86 & 895 \\
\hline & -18.181 & -16.403 & -18.701 & -17.411 \\
\hline \multirow[t]{2}{*}{$0.5-0.7$} & 253 & 24 & 121 & 1044 \\
\hline & -18.892 & -17.646 & -19.215 & -18.467 \\
\hline \multirow[t]{2}{*}{$0.7-0.9$} & 313 & 42 & 77 & 965 \\
\hline & -19.713 & -18.468 & -19.748 & -19.138 \\
\hline \multirow[t]{2}{*}{$0.9-1.2$} & 283 & 13 & 88 & 1016 \\
\hline & -20.263 & -19.314 & -20.557 & -19.679 \\
\hline \multirow[t]{2}{*}{$1.2-2.0$} & 41 & 0 & 94 & 480 \\
\hline & -20.926 & 0 & -21.078 & -20.567 \\
\hline
\end{tabular}

In summary, using the bi-modal split of the population we find results consistent with our analysis per spectral types. However, we note that the simple rest-frame color-magnitude cut is a less precise way of selecting truly early-type galaxies, at least for $z>0.7$, since the full multi-color information is used to provide the spectral type classification.

\section{Discussion and conclusions}

The fundamental result of this investigation is to have clearly established that at least up to $z \simeq 1.2$ early-type galaxies continue to be more strongly clustered than late-type galaxies. This extends to higher redshifts earlier results obtained in the local Universe (Loveday Tresse \& Maddox 1999; Norberg et al. 2002; Zehavi et al. 2002, 2005), at redshifts $z \simeq 0.6$ (Phleps et al. 2005), and quantitatively confirms early indications at $z \simeq 1$ (Phleps \& Meisenheimer 2003).

Our specific results can be summarized as follows:

1. the clustering of sub- $L^{*}$ early-type galaxies is stronger than the clustering of late-type galaxies up to $z \simeq 1.2$; a similar behavior is obtained for "red-sequence" and "blue-cloud" galaxies selected using rest-frame colors;

2. luminous, early-type galaxies are already strongly clustered at $z \sim 1$ with $3.5 \leq r_{0} \leq 5 h^{-1} \mathrm{Mpc}$;

3 . the clustering of late-type galaxies is low at all epochs with $r_{0} \simeq 2.1-3.5 h^{-1} \mathrm{Mpc}$

4. the overall clustering of bright late-type galaxies at $z \simeq 1.4$ is relatively strong with $r_{0}=3.4 h^{-1} \mathrm{Mpc}$;

5. the bias between the early-type and late-type population is nearly constant, $b_{\text {early }} / b_{\text {late }}=1.7$ over the redshift range $0.2 \leq$ $z \leq 1.2$.

A physical interpretation of these results and comparison to local values require to keep in mind that we are working with a fluxlimited sample, and thus that we are measuring galaxies of different intrinsic luminosity at different redshifts. At $z \sim 1$ the VVDS targets early-type galaxies with $-22 \leq M_{B}(A B) \leq-19$ and an average $M_{B}(A B)=-20.3$ (see Fig. 3). Early-type galaxies observed in the 2dFGRS with similar luminosities have an observed $r_{0}=6-7.5 h^{-1} \mathrm{Mpc}$, increasing with luminosity (Norberg et al. 2002). The clustering amplitude of $\xi(r)$ for early-type galaxies has therefore evolved by no more than $70 \%$ between redshift $\sim 1$ and present.

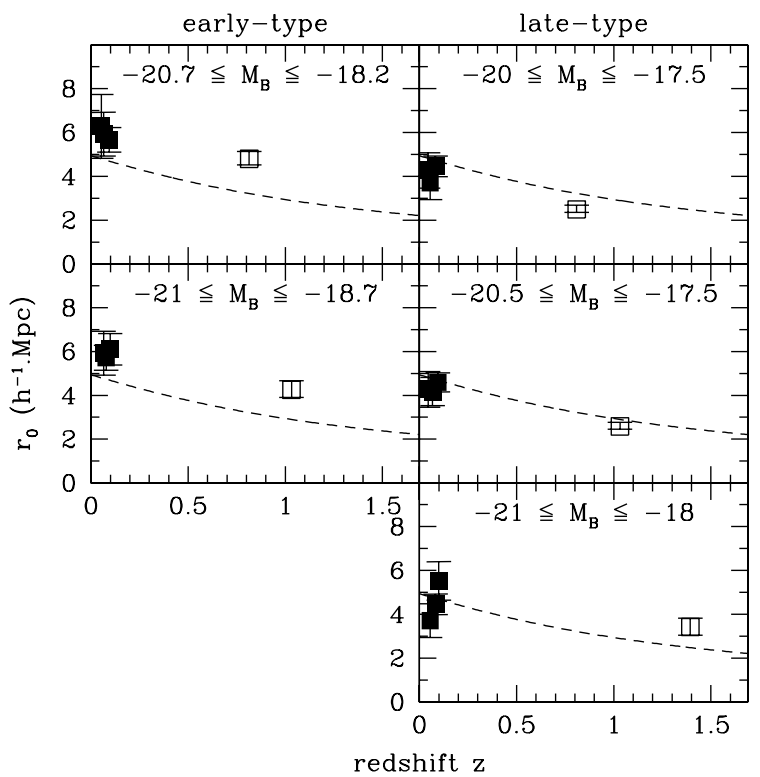

Fig. 11. Clustering length evolution for early (left panel) and late (right panel) spectral type galaxies. Measurements from the VVDS at high redshifts (open symbols) are compared to the local measurements obtained for galaxies in the same absolute luminosity ranges in the 2dFGRS (filled symbols) surveys (Norberg et al. 2002), with an evolution correction of $0.5 \mathrm{mag}$ for early types and 1 magnitude for late types at $z \sim 1$ (Zucca et al. 2005). The dashed curves show the predicted gravitational growth of the dark matter, with amplitude normalized to $\sigma_{8}=0.9$ at the current epoch.

To understand the implications of this observation, we have compared our observed evolution of $r_{0}$ to the predicted evolution of the clustering length for the dark matter in a $\Lambda$ cosmology (using the expected evolution of the variance as described in Peebles 1980). As shown in Fig. 11, if one assumes that the early-type population is simply tracing in an unbiased way the growth of clustering in the mass (as would have been the case if no evolution in number nor in luminosity occurred) their clustering would increase by a factor of $\sigma_{8}(z=1) / \sigma_{8}(z=0) \simeq 0.6$, which is close to what we see in Fig. 11. This result is in agreement with the observation of a marginal clustering evolution of early-type galaxies since $z \sim 1.5$ (Cimatti et al. 2004; Saracco et al. 2004), i.e. with the idea that the bulk of the spheroid population was already in place at these early redshifts. If a significant fraction of these galaxies was assembled and added below $z \sim 1$, we would observe not only an increase in their number density, but also a weakening of their clustering signal with respect to the underlying mass: it is natural to think that, forming typically in lower density peaks than their high-redshift companions they would in fact be less massive and less clustered.

The lower clustering level observed for early-type galaxies at $z<0.7$, is best interpreted as a luminosity effect, given the faint typical luminosity of galaxies in the first bins (e.g. $\left\langle M_{B}(A B)\right\rangle=$ -18.3 at $z=[0.2,0.5])$.

The trend observed for blue star-forming galaxies of similar luminosities is also very interesting. Below redshift $z \simeq 1$, their clustering is observed to be weak and continuously decreasing from high redshift to the present. As shown in the two right-top panels of Fig. 11, however, this is consistent with simple gravitational growth: the clustering amplitude measured at $z \sim 1$ for these galaxies is coherent with the evolution of the same structure traced by the same galaxies at $z \sim 0$. The point at $z=1.4$ may indicate that star formation activity at $z>1$ is found in 
Table 5. Measurements of the clustering length and slope for galaxy subsamples divided following the color bimodality.

\begin{tabular}{lcccccc}
\hline \hline Sample & $\begin{array}{c}\text { Number of } \\
\text { galaxies }\end{array}$ & $\begin{array}{c}\text { Redshift } \\
\text { range }\end{array}$ & $\begin{array}{c}\text { Effective } \\
\text { redshift }\end{array}$ & $\begin{array}{c}M_{B_{A B}}-5 \log (h) \\
\text { mean }\end{array}$ & $\begin{array}{c}r_{0}(z) \\
\left(h^{-1} \mathrm{Mpc}\right)\end{array}$ & $\gamma$ \\
\hline red & 270 & $0.2-0.5$ & 0.345 & -17.858 & $3.84_{-0.93}^{+0.87}$ & $1.88_{-0.16}^{+0.23}$ \\
& 277 & $0.5-0.7$ & 0.610 & -18.784 & $4.35_{-0.71}^{+0.65}$ & $1.99_{-0.18}^{+0.22}$ \\
& 355 & $0.7-0.9$ & 0.809 & -19.565 & $3.78_{-0.74}^{+0.70}$ & $1.87_{-0.22}^{+0.28}$ \\
& 296 & $0.9-1.2$ & 1.022 & -20.221 & $3.72_{-0.67}^{+0.63}$ & $1.83_{-0.32}^{+0.32}$ \\
\hline blue & 980 & $0.2-0.5$ & 0.357 & -17.521 & $2.07_{-0.39}^{+0.34}$ & $1.67_{-0.09}^{+0.14}$ \\
& 1164 & $0.5-0.7$ & 0.604 & -18.544 & $2.55_{-0.41}^{+0.39}$ & $1.68_{-0.09}^{+0.15}$ \\
& 1042 & $0.7-0.9$ & 0.811 & -19.183 & $2.18_{-0.30}^{+0.32}$ & $1.40_{-0.11}^{+0.16}$ \\
& 1105 & $0.9-1.2$ & 1.035 & -19.749 & $2.49_{-0.22}^{+0.28}$ & $1.84_{-0.10}^{+0.14}$ \\
& 574 & $1.2-2.0$ & 1.404 & -20.651 & $3.24_{-0.70}^{+0.63}$ & $1.91_{-0.18}^{+0.20}$ \\
\hline
\end{tabular}

progressively more strongly clustered galaxies than at present, i.e. plausibly in more massive galaxies in higher density peaks. In our sample, galaxies at $z=[1.2,2]$ have a mean $M_{B}(A B)=$ -20.5 and a clustering length $r_{0}=3.5 h^{-1} \mathrm{Mpc}$. Taking into account 1.5 mag of $B$-band brightening (Zucca et al. 2005) these galaxies would have today $M_{B}(A B)=-19$. Late-type galaxies with similar luminosities in the Stromlo-APM, 2dFGRS or SDSS have $r_{0} \simeq 2.9-4.2 h^{-1} \mathrm{Mpc}$ (Loveday Tresse \& Maddox 1999; Norberg et al. 2002; Zehavi et al. 2002). The clustering amplitude of late-type star-forming galaxies with comparable luminosities, therefore remains roughly constant since $z \sim 1.5$ (Fig. 11). A straightforward explanation to produce the observed result is that as redshifts get smaller, star formation is shifting to smaller-mass, less clustered galaxies.

It is difficult to compare correlation properties of samples with different mean/median magnitudes because of the well known dependence of clustering on luminosity (Guzzo et al. 1997; Budavàri et al. 2003; Zehavi et al. 2002; Pollo et al. 2006). Nevertheless, we can note that comparing our values with other clustering measurements for type- or color-selected galaxies at redshifts beyond 1.5, we find for example that star-forming LBGs at $z \sim 3$ (Adelberger et al. 2004) are even more clustered $\left(r_{0} \sim 4.5 h^{-1} \mathrm{Mpc}\right)$ than our star-forming galaxies at $z \sim 1.5$. This is consistent with the VVDS results at lower redshifts. One can speculate that star formation is moving to higher and higher density environments, when moving back in time. Similarly, red selected samples of EROs at $z \sim 1.5$ or red K-selected galaxies with $2<z_{\text {phot }}<4$ are significantly more clustered than the early type galaxies at $z \simeq 1$ in the VVDS with $r_{0}$ up to $8 h^{-1} \mathrm{Mpc}$ (Daddi et al. 2002, 2003). These galaxies seem to be populating even more extreme density peaks than those we have been studying here at $z \sim 1$.

Acknowledgements. We thank the referee, Richard Kron, for very useful comments

This research program has been developed within the framework of the VVDS consortium. This work has been partially supported by the CNRS-INSU and its Programme National de Cosmologie (France), and by Italian Ministry (MIUR) grants COFIN2000 (MM02037133) and COFIN2003 (No. 2003020150).

The VLT-VIMOS observations have been carried out on guaranteed time (GTO) allocated by the European Southern Observatory (ESO) to the VIRMOS consortium, under a contractual agreement between the Centre National de la Recherche Scientifique of France, heading a consortium of French and Italian institutes, and ESO, to design, manufacture and test the VIMOS instrument.

\section{References}

Adelberger, K. L., Steidel, C. C., Pettini, M., et al. 2005, ApJ, 619, 697 Arnouts, S., Cristiani, S., Moscardini, L., et al. 1999, MNRAS, 310, 540 Bell, E. F., Wolf, C., Meisenheimer, K., et al. 2004, ApJ, 608, 752

Benoist, C., Maurogordato, S., da Costa, L. N., Cappi, A., \& Schaeffer, R. 1996, ApJ, 472 452

Blaizot, J., Wadadekar, Y., Guiderdoni, B., et al. 2005, MNRAS, 360, 159
Bruzual, G., \& Charlot, S. 1993, ApJ, 405, 538

Budavàri, T., Connolly, A. J., Szalay, A. S., et al. 2003, ApJ, 595, 59

Carlberg, R. G., Cowie, L. L., Songaila, A., \& Hu, E. M. 1997, ApJ, 484, 538

Cimatti, A., Daddi, E., Renzini, A., et al. 2004, Nature, 430, 184

Coil, A.L., Davis, M., Madgwicket, D. S., et al. 2004, ApJ, 609, 525

Coleman, G. D., Wu, C., \& Weedman, D. W. 1980, ApJS, 43, 393

Daddi, E., Cimatti, A., Broadhurst, T., et al. 2002, A\&A, 384, L1

Daddi, E., Rottgering, H., Labbe, I., et al. 2003, ApJ, 588, 50

Davis, M., \& Peebles, J. P. E. 1983, ApJ, 267, 465

Dressler, A. 1980, ApJ, 236, 351

Fioc, M., \& Rocca-Volmerange, B. 1997, A\&A, 326, 950

Firth, A. E., Somerville, R. S., McMahon, R. G., et al. 2002, MNRAS, 332, 617

Gaztañaga, E., \& Juszkiewicz, R. 2001, ApJ, 558, L1

Giallongo, E., Salimbeni, S., Menci, N., et al. 2005, ApJ, 622, 116

Guzzo, L., Iovino, A., Chincarini, G., Giovanelli, R., \& Haynes, M. P. 1991, ApJ, 382, L5 Guzzo, L., Strauss, M. A., Fisher, K. B., Giovanelli, R., \& Haynes, M. P. 1997, ApJ, 489, 37 Ilbert, O., Tresse, L., Zucca, E., et al. (the VVDS team) 2005, A\&A, 439, 863

Ilbert, O., et al. (the VVDS team) 2005, in preparation

Iovino, A., Melnick, J., \& Shaver, P. 1988, ApJ, 330, L17

Iovino, A., Giovanelli, R., Haynes, M., Chincarini, G., \& Guzzo, L. 1993, MNRAS, 265, 21 Jenkins, A., Frenk, C. S., Pearce, F. R., et al. 1998, ApJ, 499, 20

Kaiser, N. 1984, ApJ, 284, L9

Kauffmann, G., Colberg, J. M., Diaferio, A., \& White, S. D. M. 1999a, MNRAS, 303, 188 Kauffmann, G., Colberg, J. M., Diaferio, A., \& White, S. D. M. 1999b, MNRAS, 307,529 Lahav, O., Bridle, S. L., Percival, W. J., et al. 2002, MNRAS, 333, 961

Landy, S. D., \& Szalay, A. S. 1993, ApJ, 412, 64

Le Fèvre, O., Hudon, D., Lilly, S. J., et al. 1996, ApJ, 461, 534

Le Fèvre, O., Mellier, Y., McCracken, H. J., et al. 2004a, A\&A, 417, 839

Le Fèvre, O., Vettolani, G., Paltani, S., et al. (the VVDS team) 2004b, A\&A, 428, 1043

Le Fèvre, O., Vettolani, G., Garilli, B., et al. (the VVDS team) 2005a, A\&A, 2005, 439, 845 Le Fèvre, O., Guzzo, L., Meneux, B., et al. (the VVDS team) 2005b, A\&A, 2005, 439, 877 Loveday, J., Tresse, L., \& Maddox, S. 1999, MNRAS, 310, 281

Madgwick, D. S., Hawkins, Ed., Lahav, O., et al. 2003, MNRAS, 344, 847

Marinoni, C., Le Fèvre, O., Meneux, B., et al. (the VVDS team) 2005, A\&A, 442, 801 McCracken, H. J., Radovich, M., Bertin, E., et al. 2003, A\&A, 410, 17

Mo, H. J., \& White, S. D. M. 1996, MNRAS, 282, 347

Mo, H. J., \& White, S. D. M. 2002, MNRAS, 336, 112

Norberg, P., Baugh, C. M., Hawkins, Ed. et al. 2002, MNRAS, 332, 827

Peebles, P. J. E. 1980, The Large Scale Structure of the Universe (Princeton: Princeton University Press)

Phleps, S., \& Meisenheimer, K. 2003, A\&A, 407, 855

Phleps, S., Peacock, J. A., Meisenheimer, K., \& Wolf, C. 2005 [arXiv: astro-ph/0506320]

Pollo, A., Meneux, B., Guzzo, L., et al. (the VVDS team) 2005, A\&A, 439, 887 Pollo, A., Guzzo, L., Le Fèvre, O., et al. (the VVDS team) 2006, A\&A, 451, 409 Postman, M., Franx, M., Cross, N. J. G., et al. 2005, ApJ, 623, 721

Radovich, M., Arnaboldi, M., Ripepi, V., et al. 2004, A\&A, 417, 51

Saracco, P., Longhetti, M., Giallongo, E., et al. 2004, A\&A, 420, 125 Sheth, R. K., \& Tormen, G. 1999, MNRAS, 308, 119

Shepherd, C. W., Carlberg, R. G., Yee, H. K. C., et al. 2001, ApJ, 560, 72

Smith, G. P., Treu, T., Ellis, R. S., Moran, S. M., \& Dressler, A. 2005, ApJ, 620, 78

Springel, V., White, S. D. M., Jenkins, A., et al. 2005, Nature, 435, 629

Strateva, I., Ivezic, Z., Knappet, G. R., et al. 2001, AJ, 122, 1861

van Dokkum, P. G., Franx, M., Fabricant, D., Illingworth, G. D., \& Kelson, D. D. 2000, ApJ, 541,95

Weiner, B. J., Phillips, A. C., Faber, S. M., et al. 2005, ApJ, 620, 595

Wild, V., Peacock, J. A., Lahav, O., et al. 2005, MNRAS, 356, 247

Willmer, C. N. A., Faber, S. M., Koo, D. C., et al. 2005, A\&A [arXiv: astro-ph/0506041]

Zehavi, I., Blanton, M. R., Frieman, J. A., et al. 2002, ApJ, 571, 172

Zehavi, I., Weinberg, D. H., Zheng, Z., et al. 2004, ApJ, 608, 16

Zehavi, I., Zheng, Z., Weinberg, D. H., et al. 2005, ApJ, 630, 1

Zucca, E., Ilbert, O., Bardelli, S., et al. (the VVDS team) 2005, A\&A, [arXiv:astro-ph/0506393] 